\title{
Do the global advantage and interference effects covary?
}

\author{
GHOLAMALI AMIRKHIABANI \\ Central Queensland University, Rockhampton, Queensland, Australia \\ and \\ WILLIAM J. LOVEGROVE \\ University of Wollongong, Wollongong, New South Wales, Australia
}

\begin{abstract}
The global precedence hypothesis has been operationally defined as a faster or earlier processing of the global than of the local properties of an image (global advantage) and as interference by processing at the global level with processing at the local level (global interference). Navon (1977) proposed an association between the global advantage and interference effects. Other studies have shown a dissociation between the two effects (e.g., Lamb \& Robertson, 1988). It seems that the controversy in previous research resulted from not equalizing the eccentricities of global and local properties. In the present study, the eccentricities of the two levels were equalized by using stimuli with all their elements located along their perimeters. The results of the first experiment demonstrated that although the global level was identified faster than the local level in both the central and the peripheral locations of the visual field (global advantage), the pattern of global interference varied across the visual field. Consistency of global and local levels increased the speed of processing of the local level displayed at the center of the visual field but slowed down the processing of that level at peripheral locations. The results of Experiment 2 demonstrated that it was most likely that the variation in the pattern of global interference was determined by the variable of eccentricity, rather than by the sizes of the global and local levels.
\end{abstract}

The so-called global precedence hypothesis has been studied with at least two major performance measures: the relative speed of identification and asymmetric interference. The asymmetric interference reported in the global precedence studies usually refers to a Stroop-type effect in which processing of local properties is affected by processing at the global level much more than the latter is affected by the former. There has been a controversy in the literature, however, over the association between the global/local advantage and interference effects and over its universality.

For example, Kinchla and Wolfe (1979) suggested that the temporal order of processing is not always from global to local. Rather, it varies according to the size of the compound pattern. To be able to study the effect of the size of the stimulus on processing at global and local levels, they chose to have five different sizes of stimuli, from $4.8^{\circ}$ to $22.1^{\circ}$. They mixed stimuli of these different sizes together in one block and randomly presented them to the subject. This meant that the subject was uncertain about the size and eccentricity of the incoming stimu-

Correspondence concerning this article should be addressed to $G$ Amirkhiabani, Faculty of Arts, Health and Science, Central Queensland University, Rockhampton, QLD, 4702, Australia (e-mail: a.keen@ cqu.edu.au).

- Accepted by previous editor, Myron L. Braunstein lus. ${ }^{1}$ The results of Kinchla and Wolfe's study indicated that the global level was detected faster when its size was less than $6^{\circ}-9^{\circ}$ and slower when its size was over that limit.

Navon and Norman (1983), however, noted that Kinchla and Wolfe (1979) did not equalize eccentricities of the global and the local levels. To equalize eccentricities of the two levels, Navon and Norman (Experiment 2) studied the global precedence hypothesis, using stimuli with all their elements located along their perimeters. Applying a selective attention condition, subjects were required to identify either the global or the local level of left- or right-facing large Cs made up of either left- or right-facing small Cs (Figure 1). They had two sets of stimuli-the size of the global level of one set being $2^{\circ}$, and that of the other one being $17.25^{\circ}$. There were two separate blocks for these two series of stimuli. In fact, their experiment was conducted in such a way that the subject was certain about the size and eccentricity of the incoming stimulus. The results of this experiment demonstrated that the global level was identified faster than the local components when the eccentricities of the two levels were equal. Meanwhile, it was found that, in the small visual angle condition (i.e., when the size of the global level of the stimulus was $2^{\circ}$ and, as a result, the stimulus was projected to a central location on the retina), the global level interfered with the processing of the local elements, but not vice versa. However, in the 


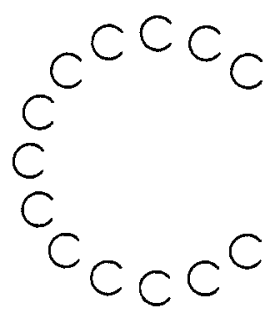

(A)

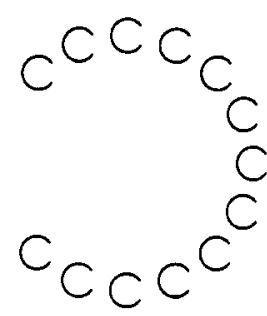

(B)

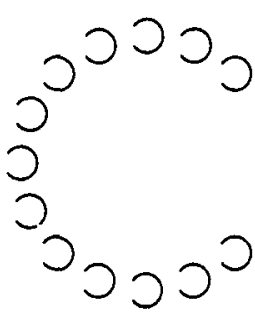

(C)

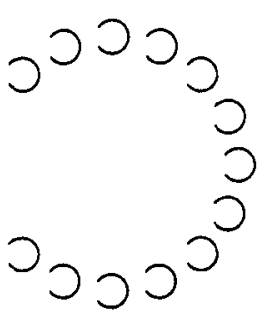

(D)

Figure 1. The set of stimuli used in this experiment. These stimuli were originally used by Navon and Norman (1983).

large visual angle condition (i.e., when the size of the global level was $17.25^{\circ}$ and, as a result, the stimulus was projected to a peripheral location on the retina), the interference effect was smaller and symmetrical.

There was a substantial difference between the study of Kinchla and Wolfe (1979) and that of Navon and Norman (1983), which makes generalization of the findings from one to the other difficult. The former study was conducted under an experimental condition in which subjects were uncertain of the size and eccentricity of the stimulus, whereas the latter was conducted in an experimental condition in which they were certain about the size and eccentricity of the stimulus. In other words, in Kinchla and Wolfe's study, stimuli of different sizes and eccentricities were mixed within a block in a random order. Thus, in each trial, the subjects had to expect stimuli of different sizes and eccentricities. In Navon and Norman's study, however, the two sizes of stimuli were presented in separate blocks. Therefore, it may be argued that the difference in the findings of these two studies was the result of the difference in the experimental conditions. One of the objectives of our study was to examine the global precedence hypothesis in a condition in which subjects were uncertain of the size and eccentricity of the stimulus.

On the basis of what has been suggested within the vision research, there may be two main interpretations of the role of eccentricity on processing at the global and the local levels. According to the first interpretation, the pattern of responses to the identification of a visual stimulus displayed across the retina may be regulated by attentional factors, such as the range effect (Humphreys, 1981; LaBerge, 1983; LaBerge \& Brown, 1986). For example, LaBerge and Brown suggested that attention, rather than sensitivity factors, dominates the processing of visual targets. That is, the decrease in acuity at peripheral locations plays a minor role, if any. In other words, according to this view, the influence of variations in eccentricity is negligible, in comparison with attentional factors. According to the second interpretation, however, visual acuity decreases with an increase in eccentricity (Amirkhiabani \& Lovegrove, 1996). The de- crease in acuity may be interpreted as being the result of a decrease in the density of cone receptors at the peripheral locations (Cornsweet, 1970). The density of the cones at a location $5^{\circ}$ away from the fovea is about $1 / 10$ of that at the foveal location, where the receptor density is maximum (Osterberg, 1935; Polyak, 1957).

The controversy over the interpretation of the role of eccentricity on processing of the visual stimulus and the lack of a systematic study of the effect of eccentricity on the processing of global and local information necessitate a comprehensive study of the role of eccentricity in the global/local interference and advantage effects. Another objective of this study is to examine the role of eccentricity in global advantage and interference effects.

The initial idea behind the global precedence hypothesis (e.g., Navon, 1977) was that reaction time (RT) advantage and asymmetric interference reflect the order of processing of global and local information. However, as Lamb and Robertson (1989) have suggested, if these two effects reflect the order of processing, they must covary in a systematic way. That means that global advantage should be associated with global interference, whereas local advantage has to be associated with local interference. However, this has not always been the case. For example, Lamb and Robertson (1989) suggested in their study that global level interfered with processing of information at the local level, whereas there was, if anything, a local RT advantage. Therefore, it might be argued that the earlier availability of global information does not necessarily justify its interference with processing at the other level.

The problem of the origin of global or local interference or advantage effects is a very important issue to investigate. However, it is not the purpose of the present study to investigate the source of these effects or to test whether the global advantage and interference effects reflect the order of processing. Readers interested in these matters may refer to Amirkhiabani and Lovegrove (1996). What we want to examine in the present study is whether, in a well-controlled experimental condition, the two effects, regardless of their origins, covary. The main objective of Experiment 1 was to investigate this latter problem. 


\section{EXPERIMENT 1}

The visual system is reported to be selectively sensitive to the spatial frequency and location of the stimulus (e.g., Cohen \& Lasley, 1974; Davis, 1981; Posner, Snyder, \& Davidson, 1980). Decreased performance was found in a detection task when subjects were uncertain about the spatial frequency or the spatial position of visual images (Davis, Kramer, \& Graham, 1983; Shapley $\&$ Lennie, 1985). To examine whether the difference between Kinchla and Wolfe's (1979) and Navon and Norman's (1983) studies originated from the difference, mentioned above, concerning certainty and uncertainty about the size and eccentricity of the incoming stimulus or, as Navon and Norman claimed, from not equalizing eccentricities of global and local levels, Experiment 1, in which the subjects were uncertain about the size and eccentricity of the incoming stimulus, was conducted. To control the confound that may be caused by the difference in the eccentricities of global and local figures, the stimuli in this experiment were chosen to be similar to those used in Navon and Norman's Experiment 2, in which the local elements were located along the perimeter of the global configuration (Figure 1). By doing this, the eccentricities of the global and the local levels were equalized. On the other hand, to be able to trace the possible effect of eccentricity on RTs to the two levels, nine series of stimuli with different sizes were projected to nine different retinal locations. Therefore, one of the objectives of this experiment was to study the global/local advantage and the interference effects in the uncertainty condition. Another objective was to investigate the effects of variations in eccentricity on the global advantage and on interference effects. More important, the aim of this experiment was to examine whether the global/local advantage and the interference effects covary across the visual field.

\section{Method}

Subjects. Seventeen right-handed undergraduate students at the University of Wollongong served as subjects. Each subject attended a single experimental session of $50-60 \mathrm{~min}$. They received either money or some course credit in return for their participation. All reported normal or corrected-to-normal vision.

Apparatus and Stimuli. The experiment was conducted in a darkened room. All the stimulus events were white on a black background. The luminance of the monitor was decreased, in order not to hurt the subjects' eyes and to decrease the effect of afterimage. To control the potential confound of afterimage, each trial was followed by a mask stimulus. The mask stimulus was a full screen of randomly located slashes and backslashes on a black background. The luminances of the test, mask, and fixation stimuli, measured by a TEKTRONIX J6526 $1^{\circ}$ Narrow Angle Luminance Probe, were approximately $2 \mathrm{~cd} / \mathrm{m}^{2}$, giving a very high contrast.

Stimulus presentation and data collection were controlled by an IBM-compatible computer. The stimuli were left- or right-facing large Cs made up of left- or right-facing small Cs (Figure 1). There were 13 small $\mathrm{Cs}$ in the perimeter of any of the large Cs. The size of the opening of all of the global or local figures was $72^{\circ}$ of its perimeter. There were nine series of stimuli, according to s1ze and eccentricity. The global configurations each had one of the following visual angle sizes: $0.5^{\circ}, 1.1^{\circ}, 2.4^{\circ}, 4.7^{\circ}, 7.2^{\circ}, 9.6^{\circ}, 12.0^{\circ}, 14.3^{\circ}$, and $16.3^{\circ}$. Since the stimuli were circular figures around the fixation point, the eccentricity of each of them was equal to half of the visual angle of the diameter (or the size) of the global configuration. The size of any of the local elements was about one eighth of that of the global configuration they belonged to. However, as was mentioned earlier, global and local levels carried the same eccentricity. Any of the stimuli illustrated in Figure 1 had an equal chance of being displayed with any of the above-mentioned sizes.

Procedure. The stimulus displays were viewed from a distance of $60 \mathrm{~cm}$, with the subject's head stabilized in a chinrest. Viewing was binocular. The task was to identify the direction of the opening of a $\mathrm{C}$ at either the global or the local level. The index fingers of the right and left hands were assigned for the right- and left-facing targets, respectively. Each subject carried out a single session, consisting of a tutoring tour, and two practice and four experimental blocks. There were 36 trials in each practice block, and 108 trials in each experimental block. One practice block and two experimental blocks were assigned to the global response, and one practice block and two experimental blocks were assigned to the local response. The results of the practice trials were not included in the analysis. The order of the practice and the experimental blocks was counterbalanced across subjects.

A trial started with the first fixation stimulus appearing in the center of the screen. The first fixation stimulus was either an " $x$ " or a " + ," measuring a visual angle of about $0.05^{\circ}$. The subjects were required to press a specially marked key with the index finger of the right hand if the first fixation stimulus was a " + " and another specially marked key with the index finger of the left hand if it was an "x." After the subject's response with either the right or the left index finger, the first fixation stimulus disappeared, and immediately after that, the test stimulus was displayed for $100 \mathrm{msec}$. Concurrent with the test stimulus, again, another " $x$ " or " + ," of the same size as the first fixation stimulus, was displayed in the center of the screen as the second fixation stimulus. The duration of the second fixation stimulus was the same as that of the test stimulus. The identities of the first and second fixation stimuli were randomly assigned by the computer. Therefore, they might be the same or different from each other. The subjects were required to notice the identity of the second fixation stimulus and were verbally questioned in about $15 \%$ of randomly selected trials. The idea behind assigning the first and second fixation stimuli was to force the subjects to fixate at the fixation point all the time and to prevent any possible eye movements (see Navon \& Norman, 1983). The test stimulus was followed by a mask, consisting of random white slashes and backslashes on a black background. The mask disappeared after the response or after a time-out of $2,000 \mathrm{msec}$. Only incorrect responses were followed by auditory feedback. The next trial started $1,000 \mathrm{msec}$ later with the first fixation point appearing on the screen. The subjects were required to respond as fast as possible, but to avoid errors. The RTs and accuracy rates to the test and the first fixation stimuli and the accuracy rates for verbal responses to the second fixation stimulus were recorded.

Key-hand assignment for the first fixation stimulus was counterbalanced across the subjects. However, in the preliminary pilot study, the subjects found responding very difficult when asked to respond to a target with an opening to the right with their left hand, and vice versa. There are similar reports by some other workers (e.g., Boer \& Keuss, 1982; Simon, Sly, \& Vilapakkam, 1981), indicating that the left-right position of the stimulus worked as an irrelevant directional cue for responding with left versus right response keys. Thus, response keys for the test stimulus were not counterbalanced across subjects. 


\section{Results}

One subject was excluded from the analysis, owing to her extremely high error rates. Trials on which responses were in error were omitted prior to analysis. The data entered into the analysis and graphed were derived from the subjects' means. A five-way repeated measures analysis of variance (ANOVA) was performed for the analysis of RTs. The factors were the first fixation stimuli (" $x$ " or " + "), the second fixation stimuli (" $x$ " or "+"), eccentricity (9 conditions), target level (global vs. local), and consistency of the levels (consistent vs. inconsistent).

Average correct RTs were grouped and are illustrated according to target level, eccentricity, and consistency in Figure 2. No significant main effects of the first and second fixation stimuli were found. An ANOVA revealed that the global configuration was identified faster than the local components $[F(1,15)=1,061.42, p<.001]$. The RTs to both global and local levels varied across eccentricity. This was reflected in the significant main effect of eccentricity $[F(8,120)=169.62, p<.001]$ and in the significant interaction of eccentricity and target level $[F(8,120)=79.75, p<.001]$. The significant interaction between eccentricity and target level also shows that the patterns of RTs to the global level, across eccentricity, were different from those to the local level.
The very slow RTs to the local levels at $0.25^{\circ}$ and $0.60^{\circ}$ seem to account for the highly reliable interaction between RTs to the two levels. Further analysis was conducted, to obtain the general linear model of the pattern of responses to the global and local levels across eccentricity. The results showed that the pattern of responses to both the global $[F(8,120)=183.84, p<.001]$ and the local $[F(8,120)=1,536.42, p<.001]$ levels best fitted with quadratic functions (Figure 2).

Although there was not an overall significant effect of consistency, the significant eccentricity $\times$ consistency $[F(8,120)=10.42, p<.001]$ and target level $\times$ eccentricity $\times$ consistency $[F(8,120)=4.45, p<.001]$ interactions suggested that the effect of consistency varied across eccentricity and target level (Figure 3 ). For eccentricities of $0.25^{\circ}, 0.6^{\circ}$, and $1.2^{\circ}$, the attended local element consistent with the unattended global configuration was identified 74,18 , and $14 \mathrm{msec}$ faster, respectively, than the attended local element inconsistent with the global configuration. For an eccentricity of $2.35^{\circ}$, RTs to the consistent and inconsistent local levels were close to each other. However, with a further increase in size and as a result of the eccentricity of the stimulus, the effect of consistency/inconsistency of the unattended global configuration on the attended local level was reversed. This time, the inconsistent local element was detected

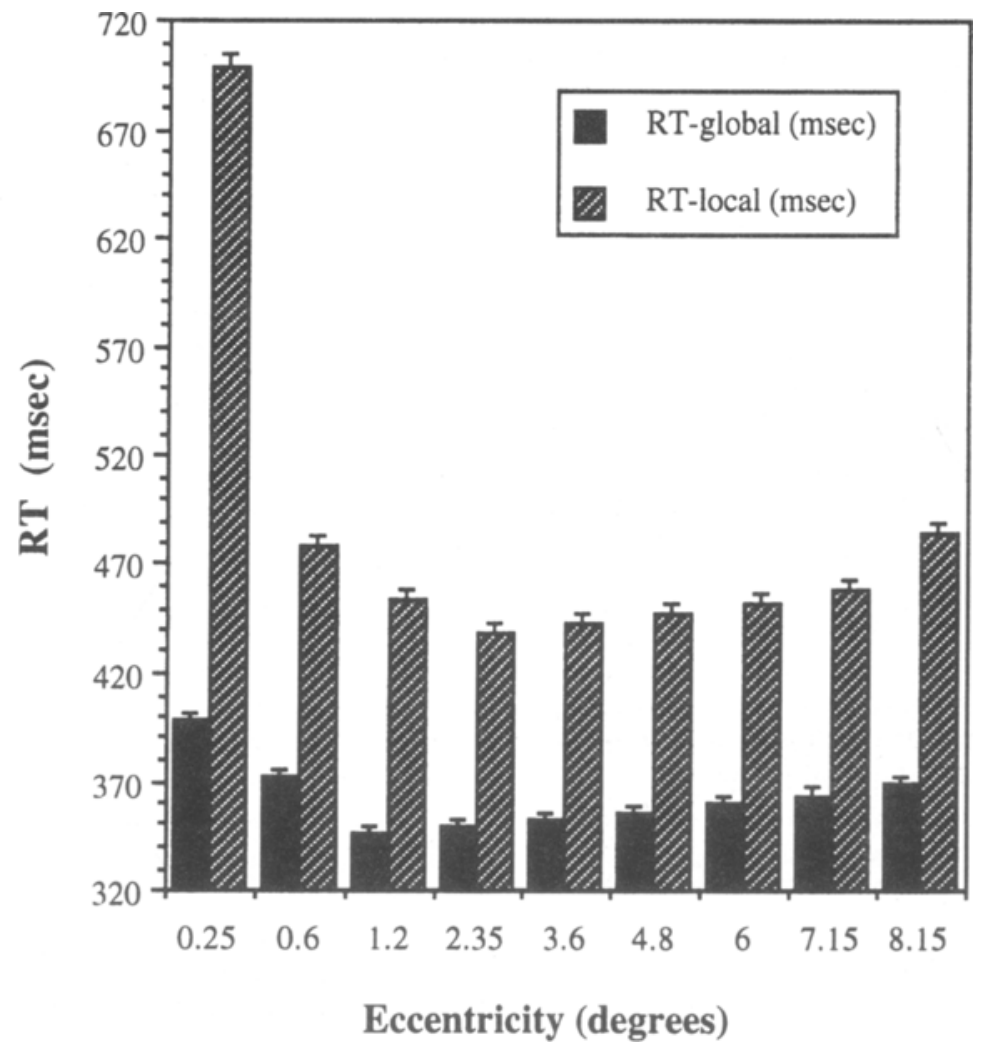

Figure 2. Mean reaction times (RTs) as a function of target level and eccentricity in Experiment 1. 


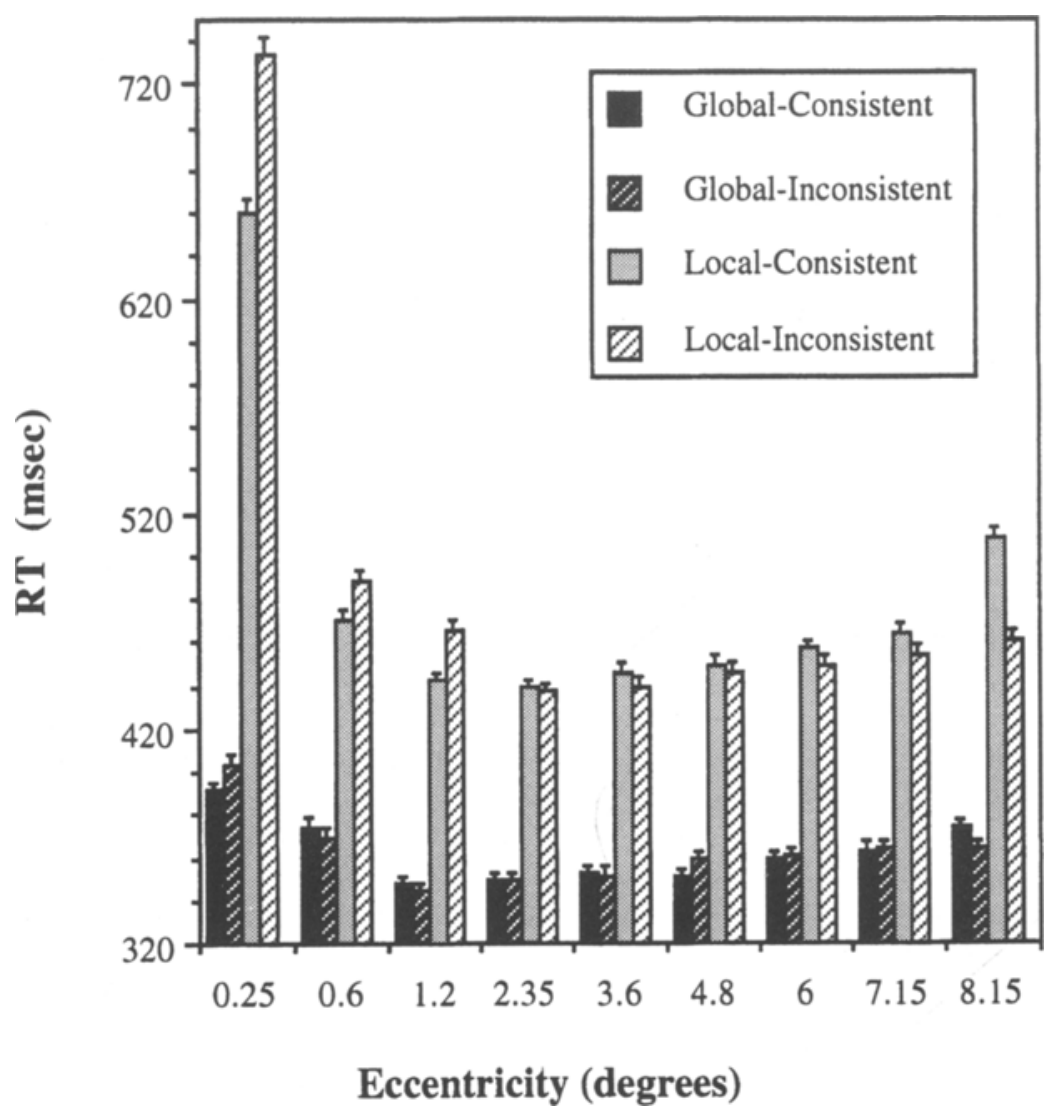

Figure 3. Mean reaction times (RTs) as a function of target level, eccentricity, and consistency in Experiment 1.

faster than the consistent one. In eccentricities of $3.60^{\circ}$, $4.80^{\circ}, 6.00^{\circ}, 7.15^{\circ}$, and $8.15^{\circ}$, these figures were $7,3,8$, 9 , and $46 \mathrm{msec}$, respectively.

On the other hand, consistency did not have a continuous and predictable effect on the identification of the global level. With eccentricities of $0.25^{\circ}$ and $4.80^{\circ}$, the attended global level, which was consistent with the identity of the local level, was detected slightly faster than the inconsistent global configuration, whereas with eccentricities of $0.6^{\circ}$ and $8.15^{\circ}$, the global configuration inconsistent with the local components was detected faster than the consistent one. The RTs to the consistent and inconsistent globals were almost the same with other eccentricities.

The consistency $\times$ eccentricity interaction was also tested separately for local and global judgments. The results showed a significant main effect of consistency $[F(1,15)=28.65, p<.001]$, a significant main effect of eccentricity $[F(8,120)=386.07, p<.001]$, and a significant interaction of eccentricity and consistency $[F(8,120)=21.43, p<.001]$ at the local level, but nonsignificant main effects and interaction of the main effects of eccentricity and consistency at the global level.
To examine whether the interference effect comes from the difference in RTs to the levels, the overall amount of global advantage was calculated by subtraction of RTs to the global and the local levels from each other for the eccentricities of 1-9. The overall amount of global advantage in eccentricities 1-9 (1 being the most central, and 9 being the most peripheral) was as follows: $301,117,108,109,91,93,93,96$, and $115 \mathrm{msec}$. As can be seen from these figures, there is a very high difference in RTs to the global and local levels in the most central eccentricity, which probably comes from the low visibility of the local level at that eccentricity. Then, the RTs decrease toward the middle of the visual field and increase again toward more peripheral locations. It does not seem that these data can explain faster RTs to inconsistent local elements at peripheral locations.

The overall accuracy proportions were $1.00(S D=$ $.00)$ to the global and $.85(S D=.15)$ to the local levels. A three-way repeated measures ANOVA was employed to study accuracy proportions. Factors were target level, eccentricity, and consistency. All the main effects were significant: target level $[F(1,15)=50.01, p<.001]$, eccentricity $[F(8,120)=16.84, p<.001]$, and consistency 
$[F(1,15)=14.17, p<.003]$. There was no significant difference between accuracy rates to the consistent and inconsistent global figures across eccentricity. However, with small eccentricities, there was a tendency for the consistent local levels to be processed more accurately than the inconsistent local levels. This trend was reversed in large eccentricity conditions. This was reflected in the significant interactions of accuracies to target level and consistency $[F(1,15)=17.21, p<.001]$, eccentricity and consistency $[F(8,120)=9.52, p<$ $.001]$, eccentricity and target level $[F(8,120)=17.73$, $p<.001]$, and target level, consistency, and eccentricity $[F(8,120)=11.90, p<.061]$. There were no other significant interactions of interest in both RT and accuracy analyses. Since all the subjects achieved a high degree of accuracy $(.98 ; S D=.01)$ in their verbal responses to the second fixation stimuli, no further analysis was carried out on this variable. The comparison of the results of latency and accuracy analysis indicated that none of the findings of RT analysis can be attributed to a speedaccuracy tradeoff.

\section{EXPERIMENT 2}

The finding of lower performance, in Experiment 1, in the consistent condition when the stimulus was displayed on peripheral locations of the retina was a curious problem. Since there was a perfect correlation between the sizes of the global shape and the local elements in Experiment 1 , it was impossible to predict from the results obtained in that experiment whether lower performance, in the consistent condition beyond the eccentricity of $2.35^{\circ}$, occurred because of variations in the size of the global level, variations in the size of the local level, eccentricity, or the combined effects of eccentricity and the size of the global or the local level. One major positive aspect of the type of stimuli used in this experiment was that, since local elements were located along the perimeter of the global configuration, it was possible to equalize (i.e., make equal) the eccentricities of the global and the local levels. In addition, the stimuli used in this experiment provided a valuable opportunity to manipulate the variables of size and eccentricity independently of each other. Considering that the experimental task was to detect the direction of the opening at either the global or the local level, it was possible to manipulate the size of the gap of the two levels without changing their eccentricity and, by doing that, to manipulate the variable of size while holding the variable of eccentricity constant. It does not seem that the size of the closed part of the Ccurve played an important role in the identification of the direction of the opening of the $C$. Therefore, it appears that speed and accuracy in performing the experimental task depend on the size of the opening of the $C$, and not on the size of the closed part of the curvature of the $\mathrm{C}$. As a result, the manipulation of the size of the gap may be assumed to be the equivalent of the manipulation of the variable of size, simply because it was the gap size that was targeted to be detected. Therefore, it seems legitimate to use the terms size and gap size interchangeably in this study. Thus, when we say that we manipulated the variable of size, we mean that we manipulated the size of the gap, and not the size of the diameters of the $\mathrm{C}$ as an element of the global configuration or the diameter of the array of Cs.

To do this, we chose three eccentricity conditions: $2.35^{\circ}$ (the turning point in the interference effect in Experiment 1 ), $0.6^{\circ}$ (below the turning point where the consistent local was detected faster than the inconsistent local), and $6^{\circ}$ (above the turning point, where the consistent local was detected slower than the inconsistent local). Therefore, we had three series of stimuli with three eccentricities. We independently manipulated the size of the gap at the global and local levels throughout all three eccentricities. The sizes of the gap of the $C$ at any of the global and local levels were one of these three angles: $24^{\circ}, 72^{\circ}$, or $120^{\circ}$. Therefore, there were $3 \times 3=9$ different combinations of gap sizes at the global and local levels in each eccentricity and, as a result, 27 combinations of gap size at the global level (3), of gap size at the local level (3), and of eccentricity (3). As was mentioned above, we manipulated the gap size of the global and local levels, and not the size of the array or the unit, simply because the task was to detect the direction of the opening of the gap of the $\mathrm{C}$ either at the global or the local level and it does not seem that the size of the array or the unit plays a role in the performance of the task.

It may be speculated that if the size (i.e., gap size, in this case) of the global level was the prime determinant of the turning point in the interference effect at the local level, variations in size (gap size) at the global level would affect the pattern of responses. If the size (gap size) at the local level was the source of variation in the interference effect, the turning point would then be dependent only on variations in size (gap size) at the local level, and not on the other two factors, size (gap size) at the global level and eccentricity. If the pattern of results in Experiment 1 was determined only by the variable of eccentricity, the turning point in Experiment 2 should only be sensitive to variations in eccentricity. Finally, there is the possibility that the turning point is the result of the combined effect of size and eccentricity. In this case, the interference effect should respond to joint variations of size and eccentricity.

\section{Method}

Most of the details of the method of this experiment were identical to those in Experiment 1, except for the following points. Twenty-three undergraduate students at Central Queensland University served as subjects. Similar to Experiment 1 , the stimuli were left- or right-facing large Cs made up of left- or right-facing small Cs. However, as was previously mentioned, the sizes of the gap at 
both the global and the local levels were one of these three angles: $24^{\circ}, 72^{\circ}$, or $120^{\circ}$. Three eccentricity conditions were chosen: $2.35^{\circ}$ (the turning point), $0.60^{\circ}$ (below the turning point), and $6.00^{\circ}$ (above the turning point). There were 13 small $\mathrm{Cs}$ on the perimeter of any of the large Cs when the size of the gap at the global level was $72^{\circ}$, 15 small Cs when the size of the gap at the global level was $24^{\circ}$, and 11 small Cs when the size of the gap at the global level was $120^{\circ}$.

As in Experiment 1, the task was to identify the direction of the opening of a C, either at the global or at the local level. Each subject carried out a single session, consisting of a tutoring tour, two practice blocks, and six experimental blocks. There were 18 trials in each practice block, and 108 trials in each experimental block. One practice block and three experimental blocks were assigned to the identification of the global level, and one practice block and three experimental blocks were assigned to the identification of the local level.

\section{Results}

A five-way repeated measures ANOVA was performed on RTs, with eccentricity $\left(0.60^{\circ}, 2.35^{\circ}\right.$, and $6.00^{\circ}$ ), target level (global and local), consistency of global and local levels (consistent and inconsistent), gap size of global level $\left(24^{\circ}, 72^{\circ}\right.$, and $\left.120^{\circ}\right)$, and gap size of local level $\left(24^{\circ}, 72^{\circ}\right.$, and $\left.120^{\circ}\right)$ as the main factors. Trials on which responses were in error were omitted prior to analysis. Main effects of target level $[F(1,22)=$ $13,393.85, p<.001]$, eccentricity $[F(2,44)=187.62$, $p<.001]$, consistency $[F(1,22)=19.13, p<.001]$, gap size at the global level $[F(2,44)=30.22, p<.001]$, and gap size at the local level $[F(2,44)=379.56, p<.001]$ were significant. A separate ANOVA was conducted on the first (" $x$ " or "+") and second (" $x$ " or " +") fixation stimuli. The results did not indicate any significant main or interaction effects.

Average correct RTs were grouped and are illustrated according to target level and eccentricity in Figure 4. The results indicated that the global level was identified faster than the local level at all three eccentricities. RTs at both the global and the local levels were faster with the medium eccentricity than with the small or the large ones. This was reflected in the significant main effect of eccentricity and in the interaction of target level and eccentricity $[F(2,44)=107.46, p<.001]$. This finding was consistent with the quadratic functions to global and local levels reported in Experiment 1. Although there was a significant main effect of consistency, further analysis indicated that there was not a significant difference between RTs to consistent and inconsistent global

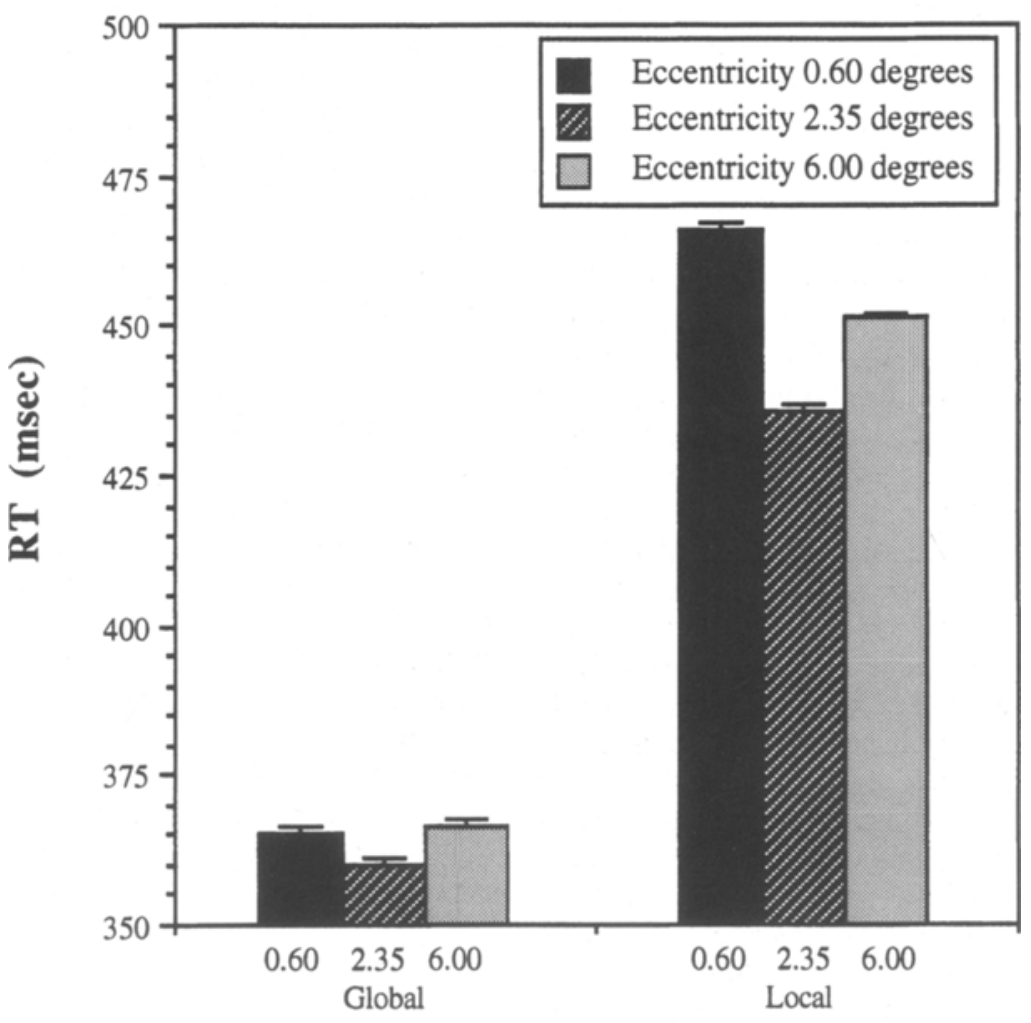

Target Level

Figure 4. Mean reaction times (RTs) as a function of target level and eccentricity in Experiment 2. 


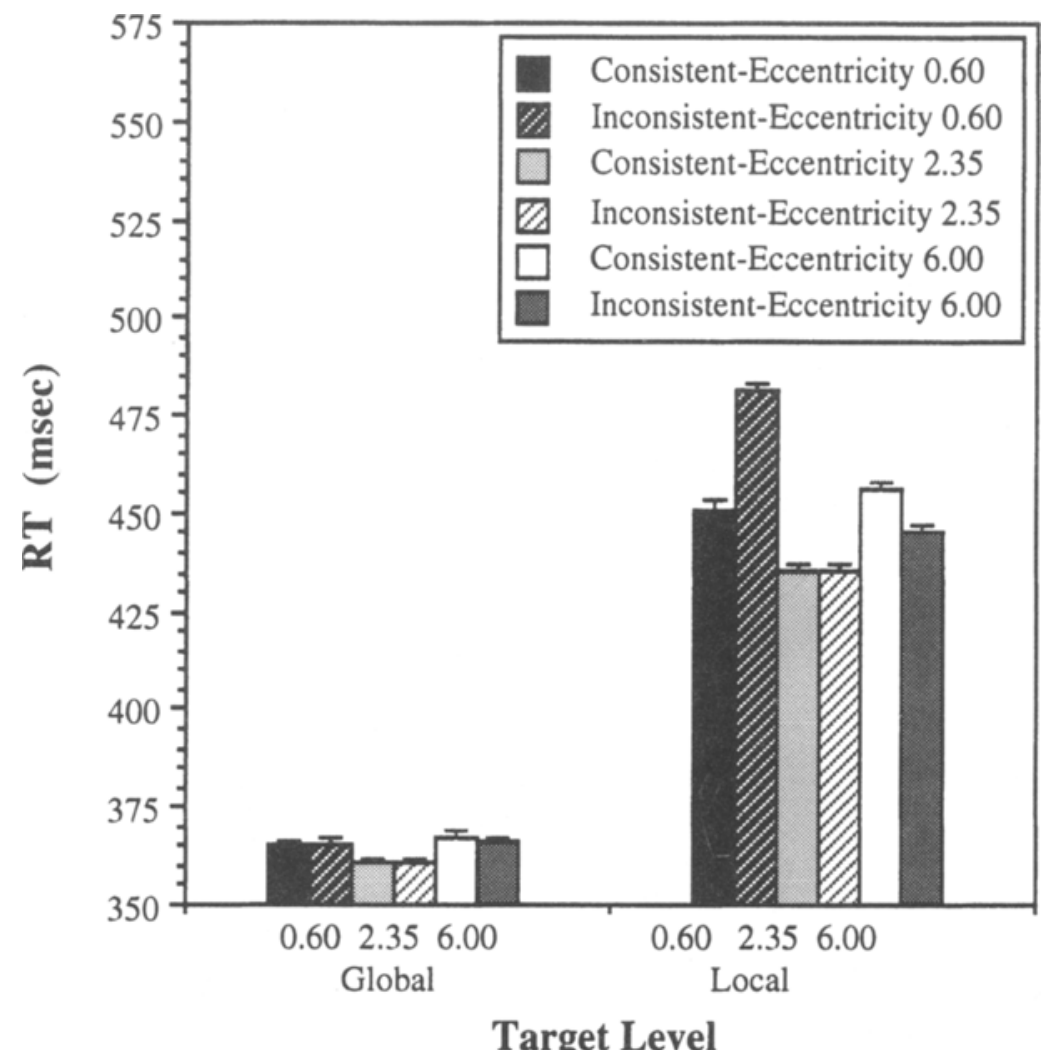

Figure 5. Mean reaction times (RTs) as a function of target level, consistency, and eccentricity in Experiment 2.

levels, but consistent locals were in general identified faster than inconsistent locals. This was reflected in the significant interaction of target level and consistency $[F(1,22)=19.87, p<.001]$. Further investigation revealed that consistency was not a continuous effect across eccentricity. Consistent locals were detected faster than inconsistent locals in small eccentricity conditions, but they were detected slower in large eccentricity conditions (Figure 5). This was reflected in the significant interaction of target level, eccentricity, and consistency $[F(2,44)=59.61, p<.001]$. There was not any significant difference between RTs to consistent and those to inconsistent local components at medium-sized eccentricity. This trend was exactly the same when the data for the local elements were broken down according to sizes (i.e., gap sizes) of global and local levels (Figures 6 and 7). This was reflected in the significant interaction of target level, eccentricity, and gap size at the global level $[F(4,88)=5.05, p<.001]$ and in the significant interaction of target level, eccentricity, and gap size at the local level $[F(4,88)=17.95, p<.001]$. These results revealed that it was the variation in eccentricity that was the prime determinant of the lower performance at the inconsistent level with the peripheral eccentrici- ties. The patterns of results did not show any sensitivity to variations in sizes (gap sizes) at global and local levels.

The results also revealed that the global configuration with a gap of $72^{\circ}$ in its parameter was identified faster than the global configuration with a gap of $24^{\circ}$ or $120^{\circ}$. This was reflected in the significant main effect of gap size at the global level and in the significant interaction of gap size at the global level and eccentricity $[F(4,44)=$ $3.2, p<.05]$. Concerning the size of the gap of local components, the subjects had difficulty detecting a local gap size of $24^{\circ}$, but there was not a significant difference between RTs to local gap sizes of $72^{\circ}$ and $120^{\circ}$. This was revealed in the significant main effect of gap size at the local level and in the significant interaction of gap size at the local level and eccentricity $[F(4,44)=14.5, p<$ $.001]$. Optimum gap size of local and global figures was probably related to their visibility and goodness of the shapes.

The magnitude of the interference effect was also examined as a function of gap size at the ignored level (global or local), in order to examine whether this effect (at either the local or the global level) changes with the discriminability of the ignored level. The magnitude of the interference effect was defined as the absolute value 


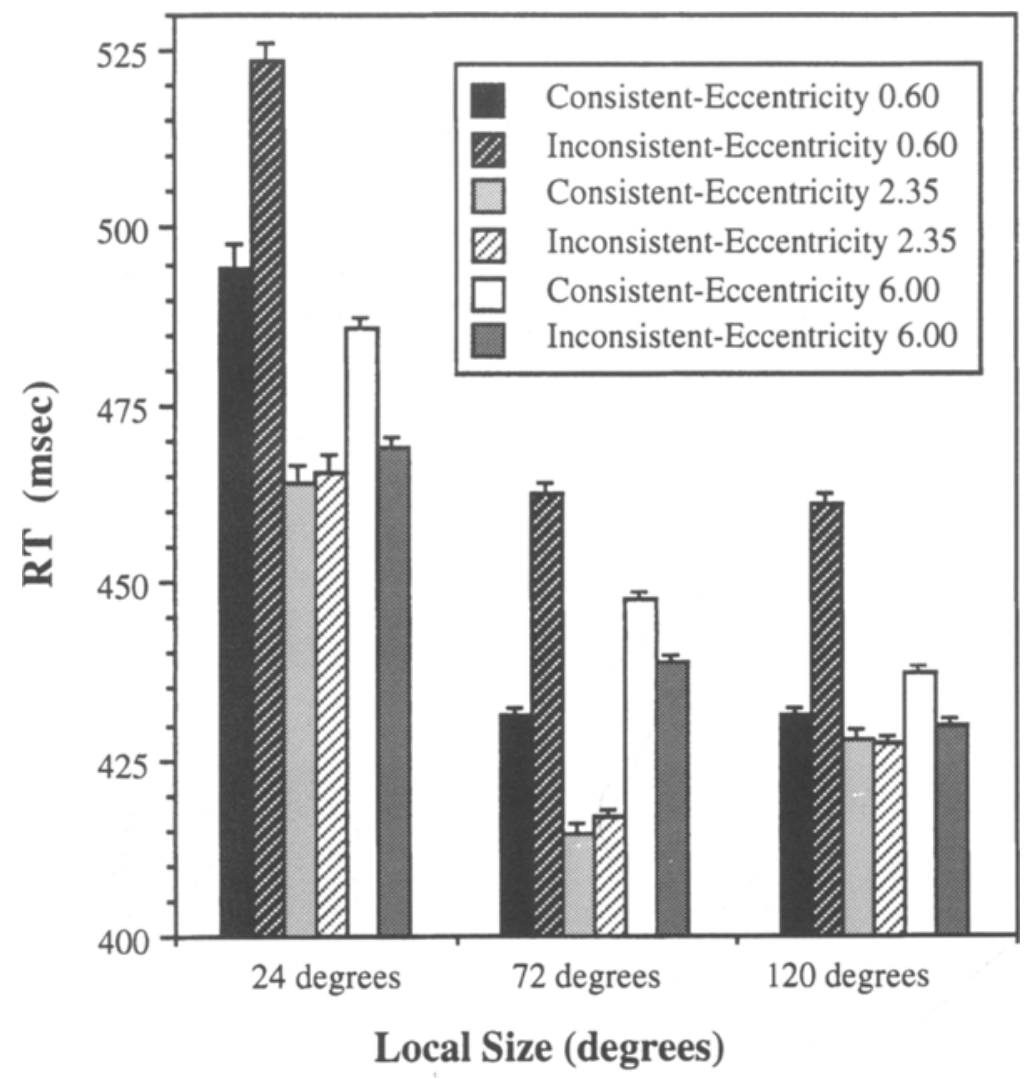

Figure 6. Mean reaction times (RTs) to local targets as a function of local size, consistency, and eccentricity in Experiment 2.

of the difference between RTs to the target level with consistent and those with inconsistent conditions. The results showed that when the size of the gap at the ignored global level was $120^{\circ}$, it had the least interference, in comparison with the condition in which the gap size was $24^{\circ}[F(1,22)=3.35, p<.05]$ and $72^{\circ}[F(1,22)=$ $3.52, p<.05]$. However, there was no significant difference between the magnitudes of interference effect when the gap size was $24^{\circ}$ and those when it was $72^{\circ}$. A similar analysis was conducted on the interference of the local with the global level. No significant effect was obtained. A further analysis was conducted for the magnitude of the interference effect as a function of gap size at the ignored level and eccentricity, but no distinct and continuous patterns were obtained.

Accuracy rates were analyzed in a similar manner to RTs. The comparison of the results of latency and accuracy analyses indicated that none of the findings of RT analysis can be attributed to a speed-accuracy tradeoff. Since all the subjects achieved a high degree of accuracy $(.99 ; S D=.01)$ in their verbal responses to the second fixation stimuli, no further analysis was carried out on this variable.

\section{Discussion}

Global advantage. The present study failed to find a relationship between retinal size of the stimulus and global advantage, in the sense proposed by Kinchla and Wolfe (1979) and some other researchers (Antes \& Mann, 1984; Lamb \& Robertson, 1989). Using stimuli with either inconsistent or consistent levels, it was found that, in the uncertainty experimental condition, the global level was identified faster than the local level across all sizes and eccentricities. Therefore, it may be concluded that there was not a difference between the results of Kinchla and Wolfe's study and those of Navon and Norman's (1983) study because there was uncertainty as to the size and eccentricity of the incoming stimulus in the first study. This difference, more than likely, was caused by not equalizing the eccentricities at the global and local levels, in the former study.

Meanwhile, the patterns of responses in the present study indicated that, with the proportional enlargement of the size and eccentricity of the stimulus, RTs to both global and local levels decreased, up to a certain size and eccentricity. Beyond this point, further increases in the size and eccentricity of the stimulus were associated with increases in RTs to both levels. These findings suggested that there is an optimum retinal location, around an eccentricity of $2.35^{\circ}$, at which the images of both global and local levels are processed faster than at other locations, when the size and eccentricity of the stimulus are proportionally enlarged. This finding may have clinical and educational implications. 


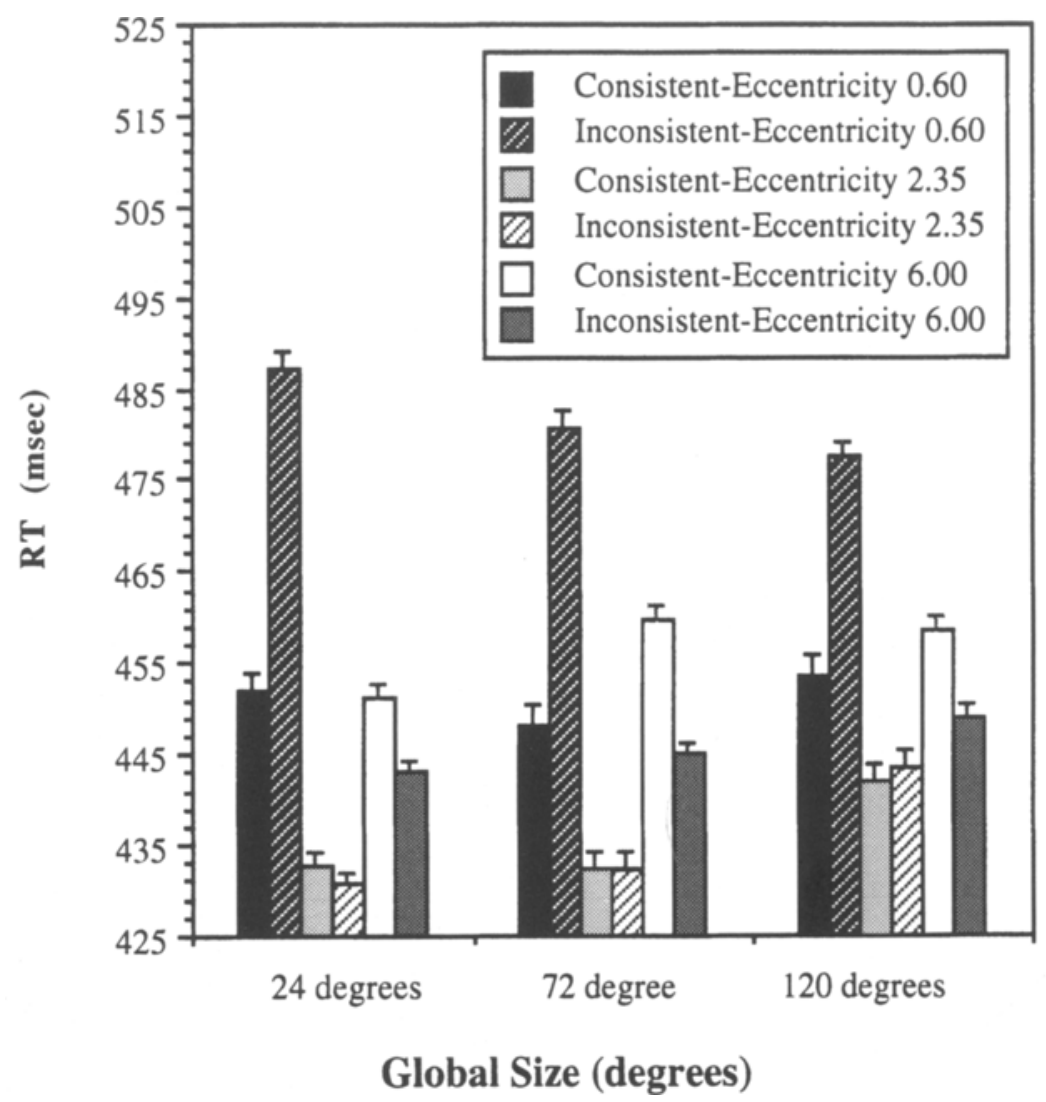

Figure 7. Mean reaction times (RTs) to local targets as a function of global size, consistency, and eccentricity in Experiment 2.

Interference effect. Generally, it appears that, with the exception of Navon and Norman's (1983) and Amirkhiabani and Lovegrove's (1996) studies, other researches did not enforce a control on the eccentricities of the global and local levels. The present experiment not only controlled this confound, but also is the only study in which the interference effect across a broad range of size and eccentricity was examined. The results demonstrated that the local level did not have a significant interference with processing at the global level across eccentricities. On the other hand, although the processing of the local level was influenced by the unattended global level, the direction of this effect changed with proportional increases in the size and eccentricity of the two levels in Experiment 1. When a small stimulus was displayed at the central eccentricities, its local elements were detected faster when they were consistent with the global configuration than when they were inconsistent. However, when a large stimulus was displayed at the peripheral eccentricities, the local components consistent with the global configuration were detected slower than those inconsistent with the global configuration.

The results of Experiment 2 showed that, in general, a consistent local level was identified faster than an inconsistent local level. Again, similar to the results of Ex- periment 1 , the effect of consistency was not uniform across eccentricities. The consistent local level was identified significantly faster at central eccentricities and slower at peripheral eccentricities, in comparison with the inconsistent local level. Further analysis confirmed that faster RTs to consistent locals at central eccentricities and to inconsistent locals at peripheral eccentricities were not determined by the size (gap size) of the global or the local levels or by their interaction. On the basis of the findings of the present study, eccentricity is the best candidate for causing such a variation in the consistency effect.

On the basis of the data presented above, it is impossible to answer the question of whether the interference effect comes from the faster RTs to the global than to the local level, mainly because faster RT to the global level does not necessarily justify its interference with the processing of the local level, and the so-called global interference effect changes its direction across the retina. Also, it was not the main objective of this study to find the origin of these effects. By asking the question of whether the global advantage and the interference effects covary, we wanted to see whether global interference occurs when there is a global advantage. The results are more complicated than we had expected. It was found 
that although global advantage was associated, or covaried (we use these two terms interchangeably), with global interference, in the sense that consistency/inconsistency of the levels affected the local and not the global level, the two effects did not covary in the sense that global advantage sometimes was associated with a decrease and at other times with an increase in RTs to the local level. Perhaps the fact that consistency/inconsistency of the two levels had an effect on the local and not on the global level (either an increase or a decrease in the speed of processing of the consistent, in comparison with the inconsistent, local) shows that the two effects (advantage and interference) covary. However, the direction of the interference effect varies across the visual field. This change in direction of the interference effect does not seem to come from the difference in speed of processing of the two levels. In answer to the question of whether global interference and advantage effects covary, we prefer the second explanation and would answer that they do not.

Perhaps the most important finding of the present experiment is that there was a discontinuity in the interference effect and that this discontinuity was determined by eccentricity. A comparison of the patterns of responses to the consistent and to the inconsistent stimuli at the global and local levels of the present study and the findings of other studies suggest that the global advantage effect is not always associated with a Stroop-type interference. More specifically, with the type of compound patterns used in our study, a distinct variation in the interference effect was observed with the manipulation of eccentricity.

In conclusion, the present work should be considered to be a reminder of the difficulty in attributing findings of temporal global/local precedence to the asymmetric interference hypothesis. Although it is tempting to generalize from the results of this experiment, there is simply insufficient data to do so. For example, compare the results of this study with Navon and Norman's (1983) findings. Although the results for the small visual angle condition (eccentricity of $1^{\circ}$ ) of Navon and Norman's Experiment 2 were the same as those for the closest eccentricity in Experiment 1 (eccentricity of $1.2^{\circ}$ ), their results demonstrated facilitation in RTs of the consistent condition for both the global and the local levels at an eccentricity of $8.5^{\circ}$. The results of the present study, however, showed that the RTs, in the inconsistent condition, to the local level at an eccentricity of $8.15^{\circ}$ were faster than those in the consistent condition. This may be because our study was conducted under an experimental condition in which there was uncertainty about the size and eccentricity of the stimulus, whereas Navon and Norman's study was conducted under a certainty condition. Another difference between the two studies was in the control of central fixation, which was probably handled better in our study than in Navon and Norman's.

This study did not attempt to answer the question of why the global level is faster than the local. The fact that the RT to the local level in the largest array (eccentricity of 8.15) was almost the same as the RT to the global level of the array displayed at an eccentricity of $1.2^{\circ}$ but that the RT to the detection of the local level was much slower than the RT to the global level of the same size projected to a central location clearly demonstrates that size cannot be considered to be the prime determinant of the global advantage effect. Interested readers on the origin of the global advantage are referred to an article by Amirkhiabani and Lovegrove (1996).

Without further experimentation, using other types of compound patterns, the present findings cannot be interpreted as firm conclusions. However, this study is the first to examine the consistency effect across a wide range of size and eccentricity, and it indicates that the interference effect is determined by the eccentricity, rather than by the size (gap size), of the global or the local level. Meanwhile, it has shown that the global advantage is a better candidate than the interference effect as a measure of the global precedence hypothesis.

\section{REFERENCES}

Amirkhiabani, G., \& Lovegrove, W. J. (1996). Role of eccentricity and size in the global precedence effect. Journal of Experimental Psychology: Human Perception \& Performance, 22, 1434-1447.

ANTES, J. R., \& MANN, S. W. (1984). Global-local precedence in picture processing. Psychological Research, 46, 247-259.

BoeR, L. C., \& Keuss, P. J. G. (1982). Global precedence as a postperceptual effect: An analysis of speed-accuracy tradeoff functions. Perception \& Psychophysics, 31, 358-366.

COHEN, T. E., \& LASLEY, D. J. (1974). Detectability of a luminance increment: Effect of spatial uncertainty. Journal of the Optical Society of America, 64, 1715-1719.

Cornsweet, T. N. (1970). Visual perception. New York: Academic Press.

DAvis, E. T. (1981). Allocation of attention: Uncertainty effects when monitoring one or two visual gratings of noncontiguous spatial frequencies. Perception \& Psychophysics, 29, 618-622.

Davis, E. T., Kramer, P., \& GraHAM, N. (1983). Uncertainty about spatial frequency, spatial position, or contrast of visual patterns. Perception \& Psychophysics, 33, 20-28.

HuMPHREYS, G. W. (1981). On varying the span of visual attention: Evidence for two modes of spatial attention. Quarterly Journal of Experimental Psychology, 33A, 17-30.

Kinchla, R. A., \& WolFE, J. M. (1979). The order of visual processing: "Top-down," "bottom-up," or "middle-out." Perception \& Psychophysics, 25, 225-231.

LABERGE, D. (1983). Spatial extent of attention to letters and words. Journal of Experimental Psychology: Human Perception \& Performance, 9, 371-379.

LABERGE, D., \& BRown, V. (1986). Variations in size of the visual field in which targets are presented: An attentional range effect. Perception \& Psychophysics, 40, 188-200.

LAMB, M. R., \& ROBERTSON, L. C. (1988). The processing of hierarchical stimuli: Effects of retinal locus, locational uncertainty, and stimulus identity. Perception \& Psychophysics, 44, 172-181.

LAMB, M. R., \& RoBERTSON, L. C. (1989). Do response time advantage and interference reflect the order of processing of global- and locallevel information? Perception \& Psychophysics, 46, 254-258.

Navon, D. (1977). Forest before trees: The precedence of global features in visual perception. Cognitive Psychology, 9, 353-383.

Navon, D., \& Norman, J. (1983). Does global precedence really depend on visual angle? Journal of Experimental Psychology: Human Perception \& Performance, 9, 955-965.

OSTERBERG, G. (1935). Topography of the layer of rods and cones in the human retina. Acta Ophthalmologica, 6 (Suppl.), 11-97. 
Polyak, S. L. (1957). The vertebrate visual system. Chicago: University of Chicago Press.

Posner, M. I., SNyder, C. R., \& DAvidson, B.-J. (1980). Attention and the detection of signals. Journal of Experimental Psychology: General, 109, 160-174.

ShaPley, R., \& LeNNIE, P. (1985). Spatial frequency analysis in the visual system. Annual Review of Neuroscience, 8, 547-583.

Simon, J. R., Sly, P. E., \& VilapakKam, S. (1981). Effect of compatibility of S-R mapping on reactions toward the stimulus source. Acta Psychologica, 47, 63-81.

\section{NOTE}

1. Eccentricity refers to the relative location of a visual object from the viewer's fixation point. The eccentricity of a point within the visual field that is located on a straight surface perpendicular to the line connecting the fixation point and the center of the eye lens at the fixation point can be expressed in terms of a visual angle, where the vertex is at the center of the viewer's eye lens, one of its sides passes through the point, and the other passes through the fixation point. The extension of the side that bisects the fixation point goes through the center of the fovea, and the extension of the other side creates the image of the point on the retına. The eccentricities of two visual objects located on a surface perpendicular to the line connecting the fixation point and the center of the eye lens at the fixation point will be equal if the two points are at an equal distance from the fixation point.

(Manuscript received February 8, 1996; revision accepted for publication July 27,1998 .) 